\title{
Self-assembly of shape-controlled nanocrystals and their in-situ thermodynamic properties
}

\author{
Z.L. Wang *, J.S. Yin \\ School of Materials Science and Engineering, Georgia Institute of Technology, Atlanta, GA 30332-0245, USA
}

\begin{abstract}
Size and shape selected nanocrystals behave like molecular matter that can be used as fundamental building blocks for constructing nanocrystal assembled superlattices. The nanocrystals form a new class of materials that have orders in both atomic and nanocrystal length-scales. The nanocrystals are passivated with organic molecules (called thiolates) that not only protect them from coalescence but act as the molecular bonds for forming the superlattice structure. The interparticle distance is adjustable, possibly resulting in tunable electric, optical and transport properties. This paper reviews our current progress in nanocrystal self-assembled materials and their thermodynamic properties probed by in-situ transmission electron microscopy (TEM). The studies mainly focused on size and shape-controlled nanocrystals to understand the role played by particle shape in determining their physical and chemical properties. Recent studies on photonic crystals and mesoporous materials such as silica and titania are also presented. (C) 2000 Elsevier Science S.A. All rights reserved.
\end{abstract}

Keywords: Nanocrystals; Supperlattices; Thiolates

\section{Introduction}

Nanomaterials are a new generation of advanced materials that exhibit unusual physical properties of technological importance. The unique properties of nanophase materials are determined not only by their intrinsic atomistic scale structure, but also by interparticle interaction. The role played by particle size is comparable, in some cases, to the particle chemical composition, adding another flexible parameter for designing and controlling their behavior. Size and even shape-selected nanocrystals behave like a molecular matter, and are ideal building blocks for two- (2-D) and three-dimensional (3-D) cluster self-assembled superlattice structures. The electric, optical, transport and magnetic properties of the structures depend not only on the characteristics of individual nanocrystals, but also on the coupling and interaction among the nanocrystals arranged with long-range translational and even orientational order. Research has successfully fabricated self-assembly passivated nanocrystal superlattices

\footnotetext{
* Corresponding author. Tel.: + 1-404-8948008; fax: + 1-4048949140.

E-mail address: zhong.wang@mse.gatech.edu (Z.L. Wang)
}

(NCSs) of metal [1], semiconductor [2] and oxide clusters [3], which are a new form of materials with fundamental interests and technological importance.

Studying of self-assembly of nanocrystals has been a focused area of the research in the last few years (for a review see [4]). The main objective is to synthesize and characterize the self-assembly of size- and shape-controlled nanocrystals. The authors also have been interested in the shape transformation and melting behavior of shape-controlled nanocrystals. This paper reviews the current progress in the field.

\section{Ordered self-assembly of nanocrystals}

Self-assembled arrays involve self-organization into monolayers, thin films, and superlattices of size-selected nanocrystals encapsulated in a protective compact organic coating [4]. Nanocrystals are the hard cores that preserve the ordering at the atomic scale; the organic molecules adsorbed on their surfaces serve as the interparticle molecular bonds and as protection for the particles in order to avoid direct core contact with a consequence of coalescing. The interparticle interaction can be changed via control over the length of the 
molecular chains. Quantum transitions and insulator to conductor transition could be introduced, resulting in tunable electronic, optical and transport properties.

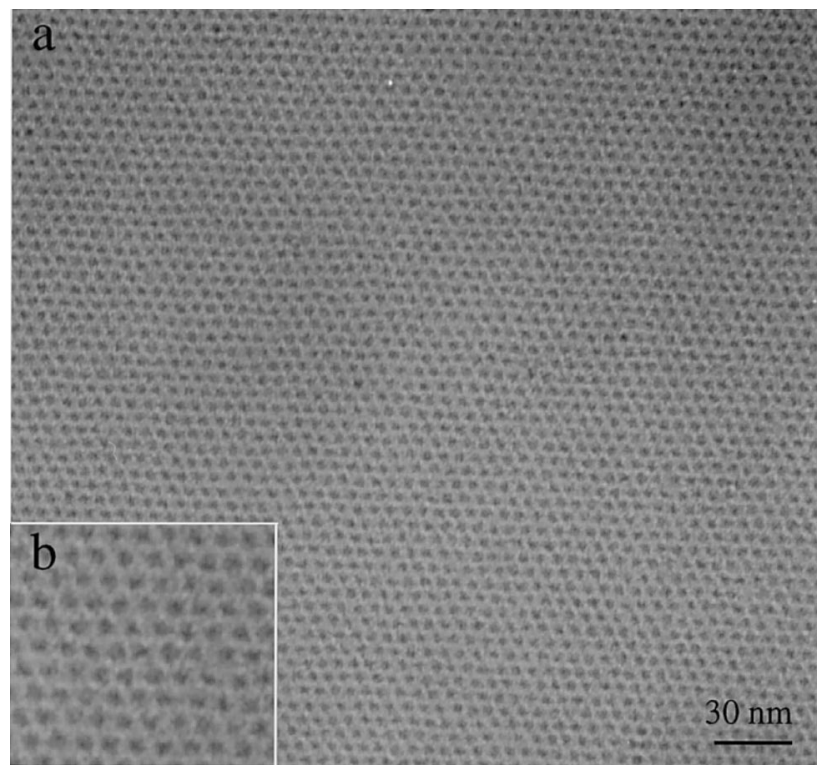

Fig. 1. (a) Transmission electron microscopy (TEM) image of monolayer self-assembled $\mathrm{Ag}$ nanocrystals with long-range translational symmetry, but only short-range orientational symmetry. (b) An enlargement of the image illustrating the projected tetrahedral shape of the nanocrystals.

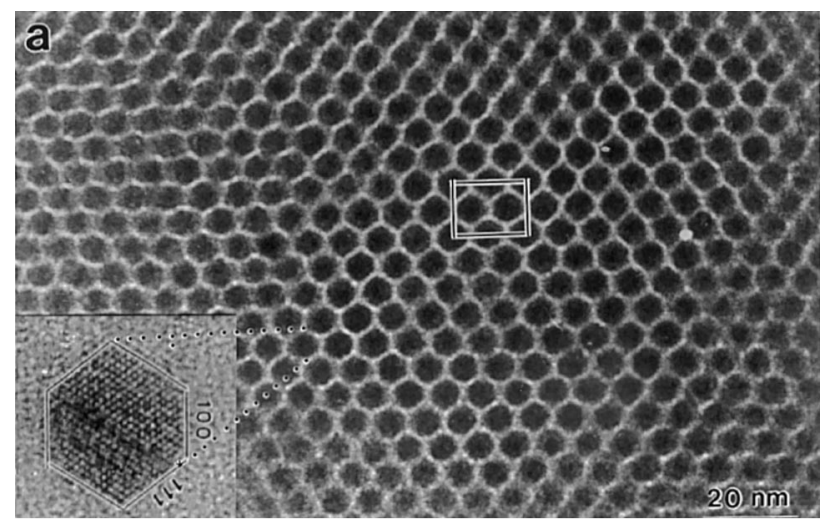

b
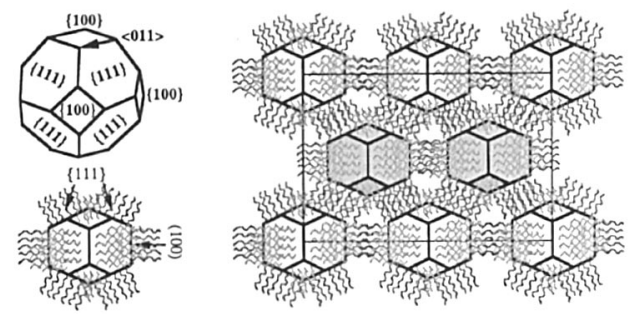

Fig. 2. (a) Self-assembly of truncated octahedral Ag nanocrystals in forming a face-centered cubic $(f c c)$ superlattice. A high-resolution transmission electron microscopy (TEM) image of a truncated octahedral Ag nanocrystal is shown in the inset, which is oriented along [110]. (b) Faceted shape of a truncated octahedra particle and its assembly in forming the $f c c$ lattice. The surface passivative molecules are also schematically drawn to illustrate the interdigitative and bundled molecular bonding.

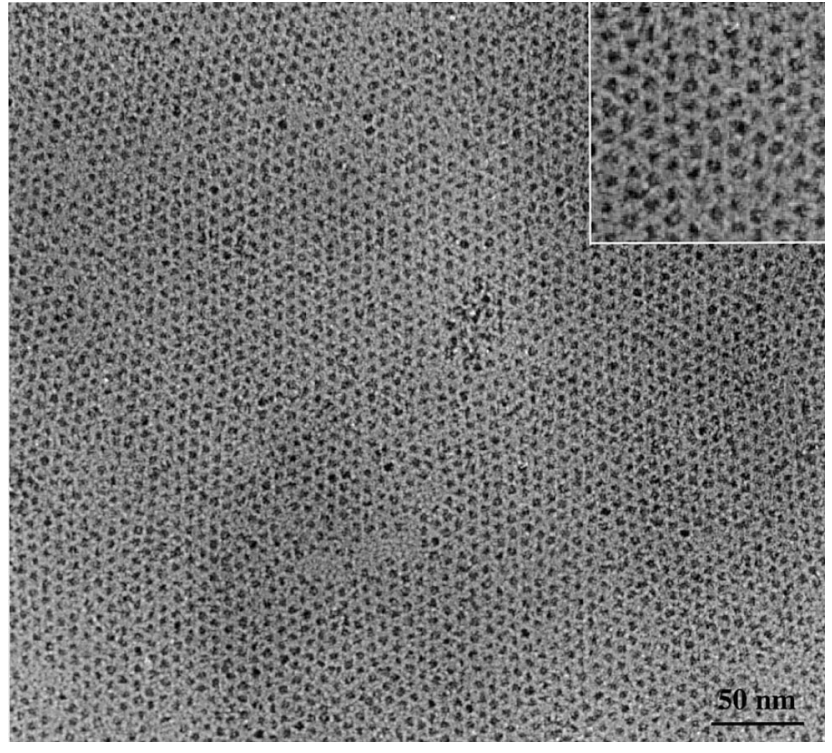

Fig. 3. Monolayer self-assembling of $\mathrm{CoO}$ nanocrystals passivated with $\mathrm{Na}(\mathrm{AOT})$. The triangle shape of the projected particle shape in Transmission electron microscopy (TEM) proves the three-dimensional (3-D) tetrahedral structure (see the inset).

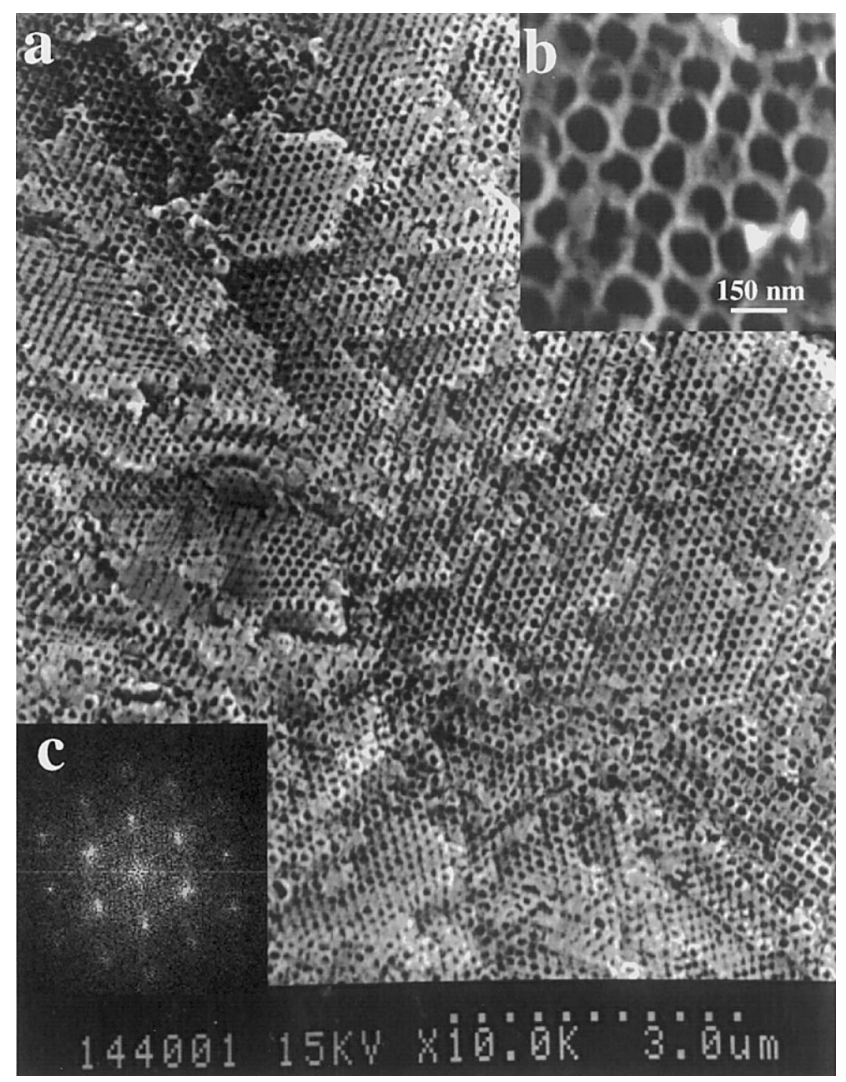

Fig. 4. (a) Scanning electron microscopy (SEM) image of an ordered hollow structure of anatase $\mathrm{TiO}_{2}$ prepared by a template-assisted technique. (b) An enlarged section of the surface. (c) A Fourier transform of the SEM to exhibit the ordering.

NCSs are characterized by unprecedented size-uniformity, translation and even orientational order, created 


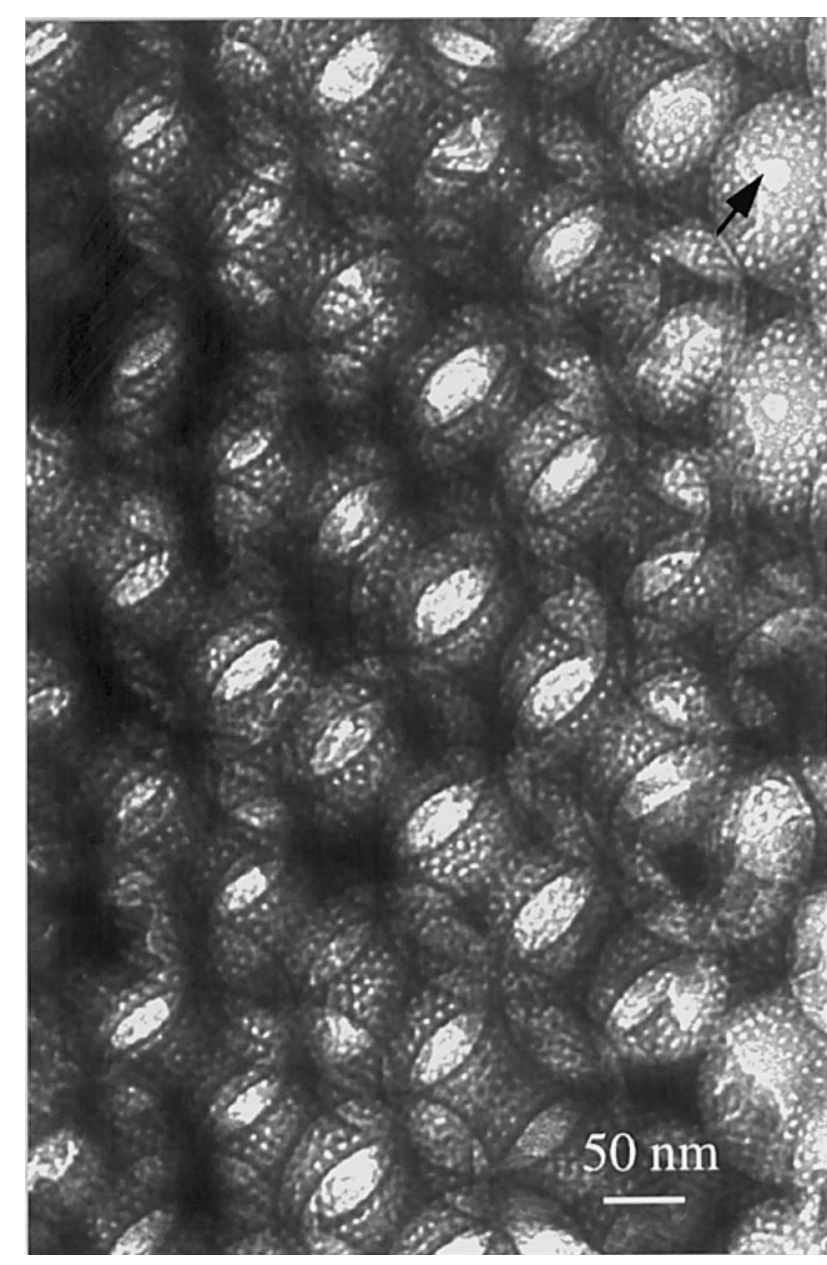

Fig. 5. Transmission electron microscopy (TEM) image of porous silica, exhibiting ordering in two length-scales: close-packed hollow spheres $(\sim 120 \mathrm{~nm})$ and self-organized nanocavities $(4-5 \mathrm{~nm})$. The arrowhead indicates a hole that interconnects the hollow spheres.

through a multistage processing strategy involving synthesis and separation of size selected nanocrystals, reversible passivation by organic monolayers, and self-assembly. Particles that can be self-assembled usually have sizes smaller than $10 \mathrm{~nm}$, it is in this size range that many exciting and unusual physical properties are enhanced. The nanocrystals are passivated by a monolayer of long-chain organic molecules, such as SR, where $\mathrm{R}=n-\mathrm{C}_{n} \mathrm{H}_{2 n+1}, \quad n=4,6,8,12 \ldots$, called thiolates.

Fig. 1a shows a transmission electron microscopy (TEM) image of monolayer assembly of Ag nanocrystals on a carbon substrate, where the 2-D superlattice is apparent. The $\mathrm{Ag}$ particles were synthesized using an aerosol technique [5] and passivated with $\mathrm{SC}_{12} \mathrm{H}_{25}$. The Ag particles have sizes of $4 \pm 0.5 \mathrm{~nm}$ and their shapes are dominated by tetrahedral (see the projected triangle shapes in Fig. 1b) [6], a four-face polyhedron enclosed by $\{111\}$ facets. Tetrahedral shape Ag nanocrystals form patterned NCSs with long-range translation order and short-range orientation order. A geometrical model has been suggested to explain the observed orientation order and the result supports that the surface adsorbed molecules are distributed on the nanocrystal surfaces, forming bundles, and the nanocrystals are assembled in such a way that the bundles tend to fill the entire space. Controlling the interparticle distance could tune the non-linear optical property [7].

The preparation and structure characterization of 3-D packing of $\mathrm{Au}$ and $\mathrm{Ag}$ nanocrystal super-structures have been reported, with an emphasis on the roles played by crystal shapes in forming the NCS [8]. In NCS of Ag nanocrystals with truncated octahedral shape, the type of orientation ordering is determined by the nanocrystal's faceted morphology, as mediated by the interactions of surfactant groups tethered to the facets on neighboring nanocrystals. Self-assembling of truncated octahedral Ag nanocrystals of $6 \pm 0.5 \mathrm{~nm}$ in core size, enclosed by eight $\{111\}$ and six $\{100\}$ facets, forms the face-centered cubic $(f c c)$ superlattice structure (Fig. 2). The particles tend to be packed faceto-face to lower the energy, the surface adsorbed

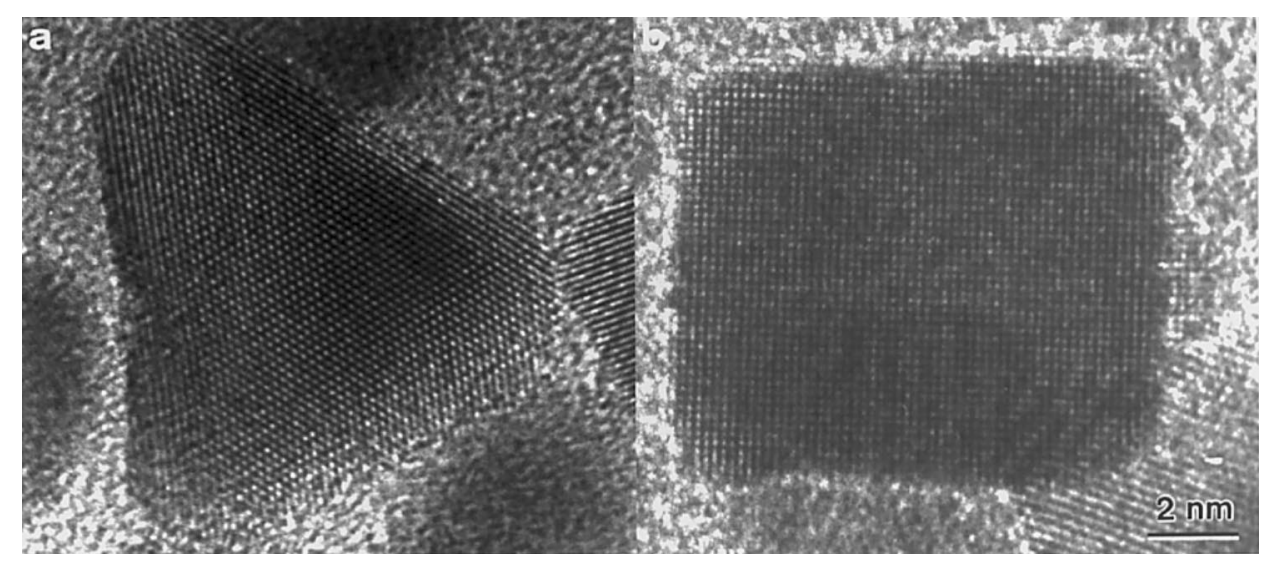

Fig. 6. (a) Tetrahedral and (b) cubic Pt nanocrystals prepared by colloidal chemistry. The images shown here present the arrangements of atomic columns as viewed along the beam direction. 


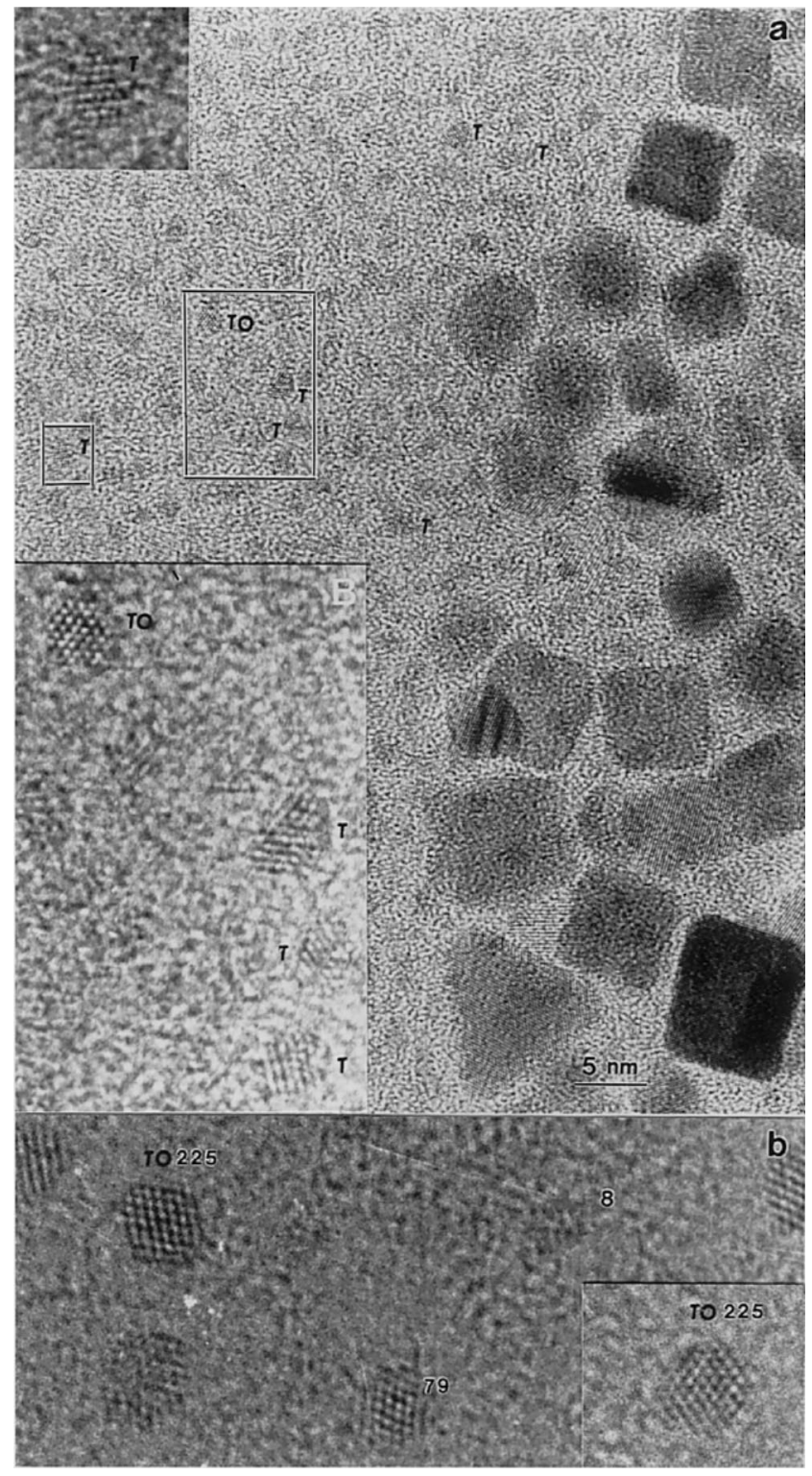

Fig. 7. (a) Transmission electron microscopy (TEM) image showing the coexistence of large and small size platinum $(\mathrm{Pt})$ nanocrystals in the solution. Enlargements of some portions of the image are inset. (b) Small Pt particles with recognizable shapes.

molecules are distributed on the facets of the particles and are self-assembled into bundled and interdigitative molecule bonds [9], which have been found to be extraordinarily strong, stabalizing the NCS to temperatures as high as $500^{\circ} \mathrm{C}$ [10]. The passivation molecules forms bundles on the surface, which have been directly observed by energy-filtered TEM [9]. NCSs formed by spherical-like (e.g. icosahedral and decahedral) Ag nanocrystals form $h c p$ superlattices [11]. Defect structures present in NCSs, including: twins, slip planes, multiply-twins and dislocations, have been studied in detail $[4,12]$.

\section{Ordered self-assembly of magnetic nanocrystals}

Patterned magnetic nanocrystals are of vital interest both scientifically and technologically. In ultra-compact information storage, for example, the size of the domain determines the limit of storage density while the sharpness of the domain boundaries is closely related to the media noise. This issue is critically important in the $300 \mathrm{Gbit} / \mathrm{in}^{2}$ information storage density predicted for the 21 st century $[13,14]$. The noise reduction can be achieved by the segregation of a non-magnetic phase at the grain boundaries, thus, the media are composed of at least two materials. The self-assembly passivated nanocrystal superlattice is a potential candidate for solving this problem, in which the passivated surfactant serves not only as an isolation layer but also as a protection layer for the nano-magnets.

For ultra-high density data storage, the most useful size range is $10-20 \mathrm{~nm}$ because the transition from ferromagnetism for large size particles to superparamagnetism for small size particles occurs at $\sim 10 \mathrm{~nm}$, and the monolayer self-assembling is the most desirable structure [15]. Monolayer self-assembling of ferromagnetic nanocrystals is difficult experimentally because of the strong agglomeration of magnetic nanocrystals due to their magnetic interaction. The ordered assembly of Co nanocrysals has been beautifully demonstrated by Sun and Murray [16].

Co ferromagnetic nanocrystals can be separated from the antiferromagnetic $\mathrm{CoO}$ and $\mathrm{Co}_{3} \mathrm{O}_{4}$ nanocrystals by a magnetic phase separation technique. The purified $\mathrm{CoO}$ nanocrystals can also form NCS with ordered structure (Fig. 3) [17]. CoO particles have a dominant tetrahedral shape of sizes $4 \pm 0.5 \mathrm{~nm}$. In-situ TEM studies of $\mathrm{CoO}$ and $\mathrm{Ag}$ NCSs have proved the structural stability up to temperatures of $\sim 500^{\circ} \mathrm{C}$ [18].

\section{Photonic crystals for optically-active devices and circuits}

Photonic crystals are materials that have a patterned periodic dielectric constant that creates an optical bandgap in the material [19]. To understand the mechanism of photonic crystals, one starts from the energy band structure of electrons in a crystalline solid. Using the Fermi velocity of the electrons in a solid, it can be found that the electron wavelength is compatible to the spacing between the atoms. Electron motion in a periodic potential results in the quantized energy levels. In the energy regions filled with energy levels, bands are formed. An energy gap between the conduction band and the valence band would be formed, which is a key factor in determining the conductivity of the solid. If the bandgap is zero, the materials are conductive; for a small bandgap, the materials are semiconductor; and the material is insulator if the bandgap is large. 
The wavelength of photons is in the order of a few hundreds of nanometers. It is necessary to artificially create a dielectric system which has periodically modulated dielectric function at a periodicity compatible with the wavelength of the photon. This can be done by processing materials that are comprised of patterned structures. As a result, photons with energies lying within the bandgap cannot be propagated unless a defect causes an allowed propagation state within the bandgap, leading to the possibility of fabricating photon conductors, semiconductors and insulators. Thus point, line, or planar defects can be shown to act as optical cavities, waveguide, or mirrors and offer a completely different mechanism for the control of light and advancement of all-optical integrated circuits. By using particle sizes in the nanometer regime with different refractive indices than the host mater- ial, these effects should be observable in the near infrared and visible spectral regions.

There are two main groups of photonic crystals. One is the self-assembly of silica spheres. The other is the ordered porous structures. Here the ordered hollow structure of titania is taken as an example [20,21]. The ordered hollow structure was made through a template-assisted technique, in which selfassembly of size selective polystyrene spheres creates an ordered template. Metal-organic solution containing the ions for synthesizing the required structure is infiltrated between the spheres. Heating the template at a higher temperature gets rid of the spheres, while the skeleton of the metal oxide still preserves. Fig. 4a is an scanning electron microscopy (SEM) image of the ordered titania structure [22]. The pore size is measured to be $90 \pm 10 \mathrm{~nm}$. A Fourier transform of
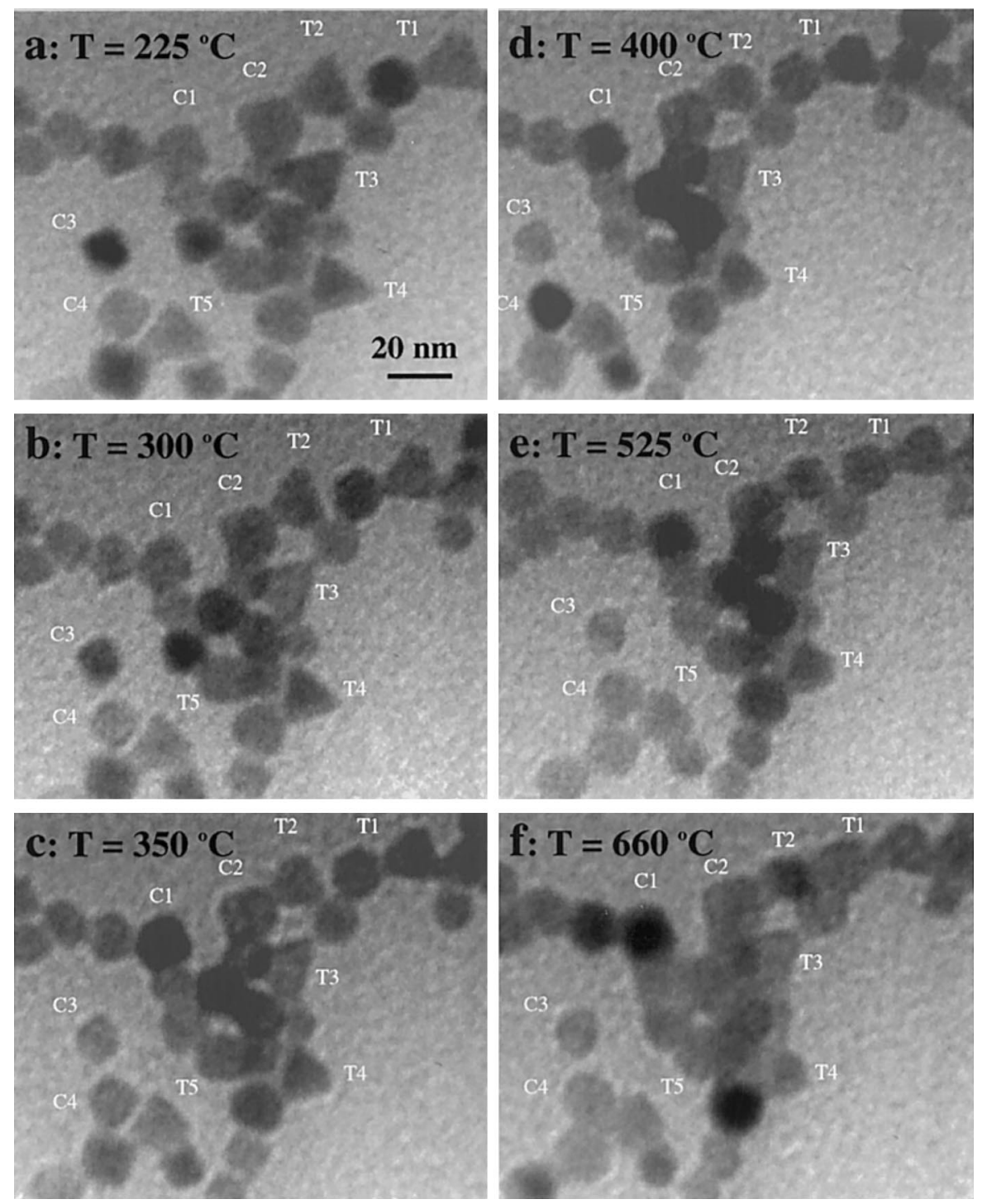

Fig. 8. Transmission electron microscopy (TEM) images recorded at different specimen temperatures, exhibiting shape transformation of platinum (Pt) nanocrystals. 

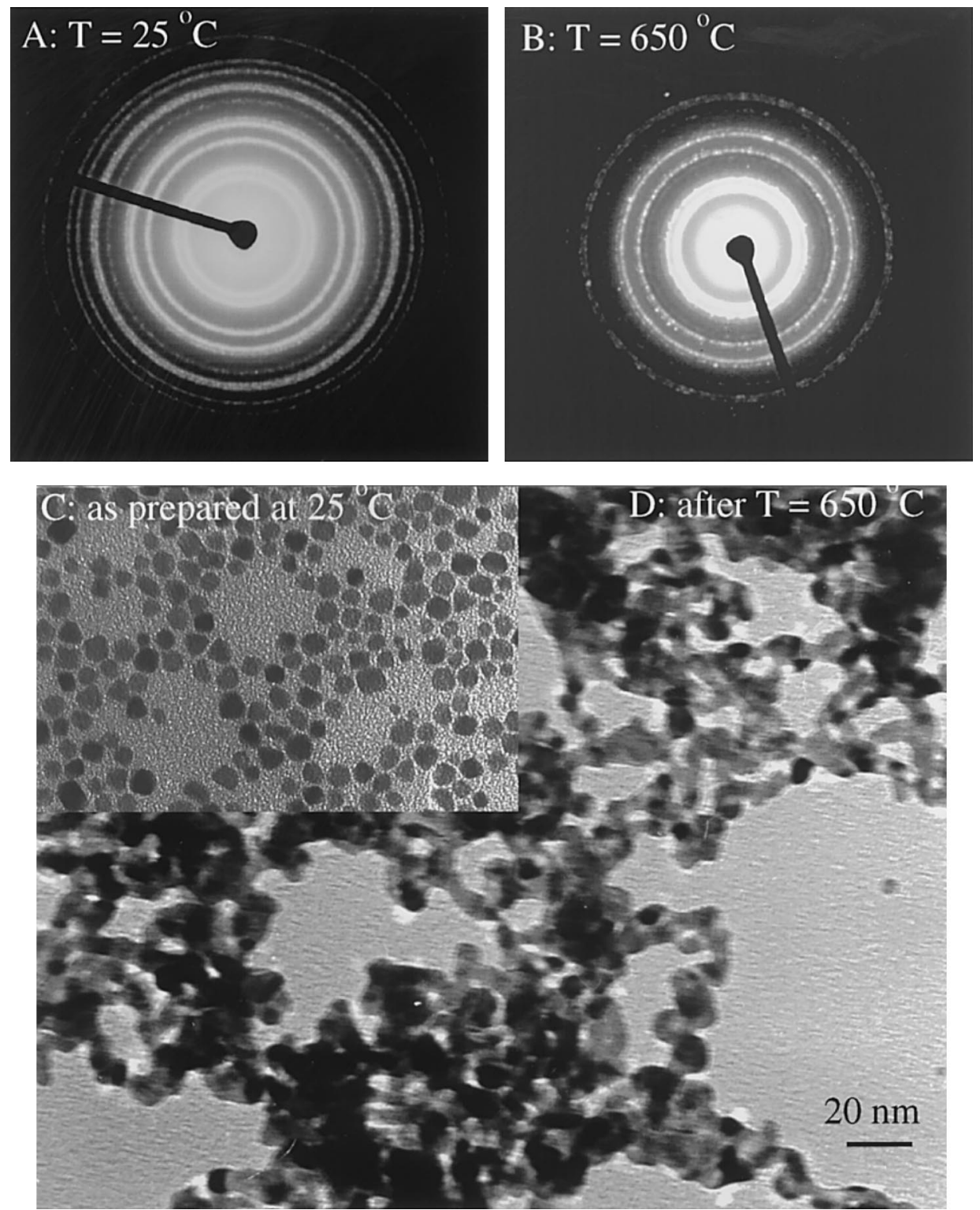

Fig. 9. (A,B) Electron diffraction (C,D) and corresponding transmission electron microscopy (TEM) images recorded at 25 and $650^{\circ} \mathrm{C}$, respectively, from platinum $(\mathrm{Pt})$ nanocrystals, showing the low melting point of nanocrystals.

the image clearly reveals the ordered self-assembly (Fig. $4 \mathrm{c})$. The pore structure is $3-\mathrm{D}$.

Anatase $\mathrm{TiO}_{2}$ nanocrystals have important applications in photocatalysts for cleaning up the organic contaminants [23] and as photoanodes in dye-sensitized solar cells [24,25]. $\mathrm{TiO}_{2}$ has a bandgap of $3.2 \mathrm{eV}$, corresponding to $338 \mathrm{~nm}$ light. When an anatase $\mathrm{TiO}_{2}$ nanocrystal is exposed to light, the UV rays cause energized electrons to break from the nanocrystal, leaving behind positively charged holes. An introduction of hole conductor filling the pores between the nanocrystals results in the flow of the holes toward the positive electrode of the photovoltaic cell. The morphology and size of the nanocrystals help the separation of the charge. Alternatively, the holes attack the hydroxide of organic compounds, resulting in their decomposition into $\mathrm{CO}_{2}$ and $\mathrm{H}_{2}$, cleaning up the environment.

\section{Mesoporous materials for low-loss dielectrics and catalysis}

Ordered self-assembly of hollow structures of silica [26], carbon [27] and titania [28,29] has drawn much attention recently because of their applications in lowloss dielectrics, catalysis, filtering and photonics. The low density of the material results in very low dielectric constant, which is a candidate for low-loss electronic devices. The large surface area of the porous materials is ideal for catalysis. The ordered hollow structure was made through a template-assisted technique as illustrated in Section 4 for titania. The structure is ordered on the length-scale of the template spheres and the pore sizes are in submicron to micron range. Alternatively, ordered porous silica with much smaller pore sizes in nanosize range $(<30 \mathrm{~nm})$, produced deliberately by 
introducing surfactant, has also been processed [20,21], in which the porosity is created by surfactants. This is the so-called mesoporous structure.

The two types of porosity have been combined into a new silica structure that has ordering and porosity on two length-scales, one is at the scale of hollow spheres created by a template of polystyrene (PS) spheres, and the other is the nanocavities created by self-assembled molecular co-polymers. This is the double length-scale ordered (DLSO) porous silica [30]. Fig. 5 gives a TEM image of the as-prepared silica porous material, clearly exhibiting ordering in the template spherical hollow scale. The size of the hollow spheres is $120 \pm 8 \mathrm{~nm}$, smaller than the size of the PS due to volume shrinkage. TEM images and diffraction patterns have shown that the packing of the hollow spheres have the hexagonal-close-packed $(h c p)\left(a_{\mathrm{h}}=120 \pm 8 \mathrm{~nm}\right)$ and the facecentered cubic $(f c c)\left(a_{\mathrm{c}}=175 \pm 10 \mathrm{~nm}\right)$ structures, and the silica is amorphous. The nanocavities formed in the walls of the shells are also revealed by the image, and
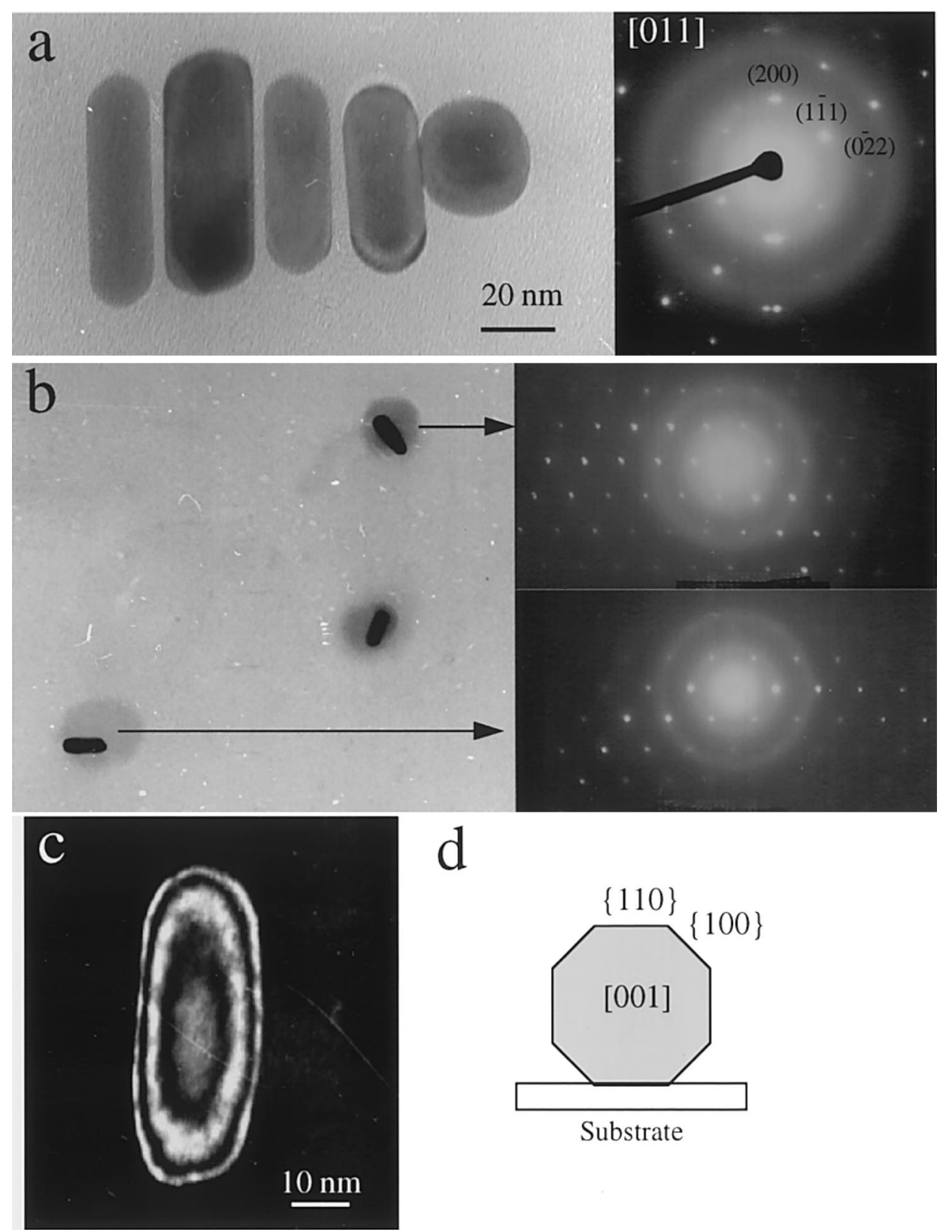

Fig. 10. (a) Transmission electron microscopy (TEM) image and the corresponding electron diffraction pattern recorded from the three Au nanorods, showing their orientations are near [011]. The facets of the rods are identified by indexing the electron diffraction pattern. (b) TEM image and the corresponding electron diffraction patterns recorded from individual Au nanorods, showing their orientations are also close to [011]. The gray mark left on the particles was due to the contamination induced by a focused electron beam while recording the diffraction pattern. (c) A dark-field TEM image of a rod recorded under the two-beam diffracting condition, exhibiting the thickness fringes across the rod. (d) A cross-section model of the nanorod showing its facet structure. 
their sizes are $4-5 \mathrm{~nm}$ and the interpore distance is $8 \pm 0.5 \mathrm{~nm}$. The synthesis technique is adequate for large-scale production. The surface areas of the silica structure has been found as high as $700-800 \mathrm{~m}^{2} \mathrm{~g}^{-1}$.

\section{Shape controlled Pt nanocrystals and their in-situ shape transformation and melting}

The catalytic activity and selectivity of nanosize colloidal platinum $(\mathrm{Pt})$ nanocrystals may depend strongly on their sizes and shapes. To determine the shape dependent catalytic behavior of Pt nanocrystals, two key steps are involved. First, shape controlled Pt nanocrystals must be synthesized at a high yield. Secondly, the passivation layer on the nanocrystal must be removed in order to measure the catalytic selectivity and activity while the particle shape is preserved. The former which has been successful demonstrated that platinum nanoparticles with a high percentage of cubic-, tetrahedral- and octahedral-like shapes, respectively, can be synthesized by a colloidal chemistry method (Fig. 6) [31,32].

To study the growth mechanism of the shape-controlled Pt nanocrystals, the smallest Pt nanocrystals that can be clearly defined using an TEM have been examined. A lower magnification image of the particles dispersed on a carbon substrate shows the co-existence of large size as well as small size particles (Fig. 7a), where the smaller particles are dominated by tetrahedra and truncated octahedra, while the large particles are dominated by cubic. This shape transformation of from tetrahedral-to-octahedral-tocubic is the result of the kinetically controlled growth [33]. Small size clusters of truncated octahedra can be imaged (Fig. 7b). The number of atoms presented in these particles may be close to 225 and 79 provided the particle shapes are perfect.

To measure the shape dependent catalytic activity and selectivity of the $\mathrm{Pt}$ nanocrystals, the capping polymer must be removed. One method could be annealing the nanocrystals supported on a substrate for evaporating the capping polymer. Several questions, however, must be considered: (1) how high does the annealing temperature need to be to remove the capping polymer while the particle shape is still preserved?; (2) to what temperature is the particle shape still stable?; (3) is there a temperature induced shape transformation due to surface diffusion?; (4) how high is the melting point of the Pt particles?; and (5) what is the effect of substrate on nanoparticle melting? These questions have not only fundamental importance, but also practical impact on the catalysis applications of the particles because the chemical reaction may take place at a higher temperature. These ques- tions have been answered using the information provided by in-situ TEM [34] (Fig. 8). The results indicate that the surface capping polymer is removed by annealing the specimen to a temperature of 180 $250^{\circ} \mathrm{C}$, while the particle shape shows no change up to $\sim 350^{\circ} \mathrm{C}$. In a temperature range of $350-450^{\circ} \mathrm{C}$, a small truncation occurs in the particle shape but no major shape transformation. The particle shape experiences a dramatic transformation into spherical-like shape when the temperature is higher than $\sim 500^{\circ} \mathrm{C}$; the macroscopic surface melting occurs at $\sim 600^{\circ} \mathrm{C}$, much lower than the melting point of bulk $\mathrm{Pt}$ $\left(1773^{\circ} \mathrm{C}\right)$.

Fig. 9A,B compares the electron diffraction patterns recorded from Pt nanocrystals at 25 and $650^{\circ} \mathrm{C}$, respectively. A sharp decrease in the intensity of the high order reflections at $650^{\circ} \mathrm{C}$ maybe due to two reasons. The occurrence of surface melting, as directly seen in the images recorded at $650^{\circ} \mathrm{C}$ (Fig. 9D), reduces the long-range ordering of the atom lattice. The increased atom vibration enhances the Debye-Waller factor, leading to the decrease of the high angle scattering. The TEM image is in favor of the interpretation of surface melting.

\section{Faceted shape of rod-like nanocrystals}

Nanocrystals can have a wide range of shapes. The recently synthesized gold nanorods by a electrochemical micelle technique [35] are rather unique [36]. The rod is bounded by facets and it is a thermodynamically un-stable crystal growth. Almost all of the $\mathrm{Au}$ nanorods are single crystalline and contain no twins or dislocations. When dispersing a liquid drop onto a flat carbon substrate, the nanorods are preferentially aligned and oriented along [110] perpendicular to the substrate (Fig. 10a). The single $\mathrm{Au}$ nanorods also prefer to orient along [110] (Fig. 9B). This is possible only if the nanorods have $\{110\}$ facets (Fig. 10b). A dark-field TEM image recorded using a $\{111\}$ reflected beam when the rod is oriented nearly parallel to the [110] direction gives the thickness fringes (Fig. 10c) owing to thickness variation across the specimen. The intervals between the fringes would have an equal distance if the nanorods were bound only by four $\{100\}$ facets. The finite width of the band at the center and its uniformity in intensity indicate that the thickness at the center region is nearly constant with a width of $\sim 5 \mathrm{~nm}$, strongly supporting the presence of $\{110\}$ facets. The axial growth direction is [001], and its cross-section is given in Fig. 10d. The presence of the $\{110\}$ high energy faces is a unique structural characteristics of the rods, possibly resulting in surface sublimation at low temperatures [37]. 


\section{Summary}

Nanomaterials are a fundamental component of nanoscience and nanotechnology in the 21 st century. Research in nanomaterials faces many challenges in synthesis, property characterization and device fabrication. The small size and large diversity in shapes of nanostructures are particularly attractive for exploring many unique and novel properties. The research has been focused on shape-controlled nanocrystals, aiming at investigating shape- or crystallographic-plane dependent properties. As a first step towards this goal, the authors have been successful in synthesis of size and shape controlled nanocrystals. This provides the fundamental for further studies on their properties and applications.

\section{Acknowledgements}

Thanks to our Georgia Tech collaborators: S.A. Harfenist, J. Petroski, T. Green, P. Poncharal, M. Mohamed, S. Link, R.L. Whetten and M.A. El-Sayed, for contributions to the work reviewed here. Thanks for the financial support of NSF grants DMR-9733160 and CHE-9727633.

\section{References}

[1] R.L. Whetten, J.T. Khoury, M.M. Alvarez, S. Murthy, I. Vezmar, Z.L. Wang, C.C. Cleveland, W.D. Luedtke, U. Landman, Adv. Mater. 8 (1996) 428.

[2] C.B. Murray, C.R. Kagan, M.G. Bawendi, Science 270 (1995) 1335.

[3] M.D. Bentzon, J. van Wonterghem, S. Morup, A. Thölén, Philos. Mag. B 60 (1989) 169.

[4] Z.L. Wang, Adv. Mater. 10 (1998) 13.

[5] M.M. Alvarez, I. Vezmar, R.L. Whetten, J. Aerosol Sci. 29 (1998) 115.

[6] Z.L. Wang, S.A. Harfenist, I. Vezmar, R.L. Whetten, J. Bentley, N.D. Evans, K.B. Alexander, Adv. Mater. 10 (1998) 808.
[7] C.P. Collier, R.J. Saykally, J.J. Shiang, S.E. Henrichs, J.R. Heath, Science 277 (1997) 1978.

[8] S.A. Harfenist, Z.L. Wang, M.M. Alvarez, I. Vezmar, R.L. Whetten, J. Phys. Chem. B 100 (1996) 13904.

[9] Z.L. Wang, S.A. Harfenist, R.L. Whetten, J. Bentley, N.D. Evans, J. Phys. Chem. B 102 (1998) 3068.

[10] S.A. Harfenist, Z.L. Wang, J. Phys. Chem. B 103 (1999) 4342.

[11] S.A. Harfenist, Z.L. Wang, M.M. Alvarez, I. Vezmar, R.L. Whetten, Adv. Mater. 9 (1997) 817.

[12] Z.L. Wang, Mater. Charact. 42 (1999) 101.

[13] Y. Nakamura, J. Magn. Soc. Jpn. 15 (1991) 487.

[14] I. Tagawa, Y. Nakamura, J. Magn. Soc. Jpn. 13 (1989) 97.

[15] J.S. Yin and Z.L. Wang, Nanostruct. Mater. 11 (1999) 845.

[16] S. Sun, C.B. Murray, J. Appl. Phys. 85 (1999) 4325.

[17] J.S. Yin, Z.L. Wang, Phys. Rev. Lett. 79 (1997) 2570.

[18] J.S. Yin, Z.L. Wang, J. Phys. Chem. B 101 (1997) 8979.

[19] J.D. Joannopoulos, P.R. Villeneuve, S. Fan, Nature 386 (1997) 143.

[20] J.E.G.J. Wijnhoven, W.L. Vos, Science 281 (1998) 802.

[21] B.T. Holland, C.F. Blanford, A. Stein, Science 281 (1998) 538.

[22] J.S. Yin, Z.L. Wang, Adv. Mater. 11 (1999) 469.

[23] C.T. Whipple, Photonics (1999) 53.

[24] B. O’Regan, M. Grätzel, Nature 353 (1991) 737.

[25] S.Y. Huang, L. Kavan, I. Exnar, M. Grätzel, J. Electrochem. Soc. 142 (1995) L142.

[26] O.D. Velev, T.A. Jede, R.F. Lobo, A.M. Lenhoff, Nature 389 (1997) 448.

[27] A.Z. Zakhidov, R.H. Baughman, Z. Iqbal, C. Cui, I. Khayrullin, S.O. Dantas, J. Marti, V.G. Ralchenko, Science 282 (1998) 897.

[28] C.T. Kresge, M.E. Leonowioz, W.J. Roth, J.C. Vartuli, J.S. Beck, Nature 359 (1992) 710.

[29] D. Zhao, J. Feng, Q. Huo, N. Melosh, G.H. Fredrickson, B.F. Chmelka, G.D. Stucky, Science 279 (1998) 548.

[30] J.S. Yin, Z.L. Wang, Appl. Phys. Lett. 74 (1999) 2629.

[31] T.S. Ahmadi, Z.L. Wang, T.C. Green, A. Henglein, M.A. ElSayed, Science 272 (1996) 1924.

[32] Z.L. Wang, T.S. Ahmadi, M.A. El-Sayed, Surface Sci. 380 (1997) 302.

[33] J.M. Petroski, Z.L. Wang, T.C. Green, M.A. El-Sayed, J. Phys. Chem. B 102 (1998) 3316.

[34] Z.L. Wang, J. Petroski, T. Green, M.A. El-Sayed, J. Phys. Chem. B 102 (1998) 6145.

[35] Y. Yu, S. Chang, C. Lee, C.R.C. Wang, J. Phys. Chem. B 101 (1997) 6661.

[36] Z.L. Wang, M. Mahamed, S. Link and M.A. El-Sayed, Surf. Sci. 440 (1999) L809.

[37] M. Mahamed, Z.L. Wang, and M.A. El-Sayed, J. Phys. Chem. A 103 (1999) 10255. 